\title{
Bayesian Inference for Heterogeneous Caprock Permeability Based on Above Zone Pressure Monitoring
}

\author{
Argha Namhata ${ }^{1,2, *}$, Mitchell J. Small ${ }^{1}$, Robert M. Dilmore ${ }^{2}$, David V. Nakles ${ }^{1}$, Seth King $^{3}$
}

1 Department of Civil \& Environmental Engineering, Carnegie Mellon University, Pittsburgh, PA 15217, USA

2 U.S. Department of Energy, National Energy Technology Laboratory, 626 Cochrans Mill Road, Pittsburgh, Pennsylvania 15236, USA

3 U.S. Department of Energy, National Energy Technology Laboratory, AECOM, 3610 Collins Ferry

Road, Morgantown, WV 26507

*Corresponding Author: Email: anamhata@andrew.cmu.edu (A. Namhata), Tel: +1 (412) 3704671.

\begin{abstract}
The presence of faults/ fractures or highly permeable zones in the primary sealing caprock of a $\mathrm{CO}_{2}$ storage reservoir can result in diffusive leakage of $\mathrm{CO}_{2}$. Monitoring of leakage requires the capability to detect and resolve the onset, location, and volume of leakage in a systematic and timely manner. Pressure-based monitoring possesses such capabilities. This study demonstrates a basis for monitoring network design based on the characterization of $\mathrm{CO}_{2}$ leakage scenarios through an assessment of the integrity and permeability of the caprock inferred from above zone pressure measurements. Four representative heterogeneous fractured seal types are characterized to demonstrate seal permeability ranging from highly permeable to impermeable. Based on Bayesian classification theory, the probability of each fractured caprock scenario given above zone pressure measurements with measurement error is inferred. The sensitivity to injection rate and caprock thickness is also evaluated and the probability of proper classification is calculated. The time required to distinguish above zone pressure outcomes and the associated leakage scenarios is also computed.
\end{abstract}




\section{INTRODUCTION}

$\mathrm{CO}_{2}$ capture and storage (CCS) is considered a promising strategy for the reduction of anthropogenic greenhouse gas emissions to the atmosphere (IPCC, 2005). However, injecting large volumes of $\mathrm{CO}_{2}$ may cause subsurface pressurization over large spatial domains, resulting in leakage that returns the injected $\mathrm{CO}_{2}$ to the atmosphere and potentially harming natural resources (e.g., groundwater resources) (Birkholzer and Zhou, 2009; Pruess, 2004). To protect the environment and public health, a comprehensive risk profile should be established for each CCS project. To monitor these risks, it is necessary to have the capability to identify and resolve the onset, location, and volume of leakage from the reservoir in a systematic and timely manner. The monitoring of pressure changes, as an indication of leakage, represents one approach to provide this information (Jung et al., 2013; Sun and Nicot, 2012). A pressure monitoring network that resolves the spatial and temporal variations of the subsurface pressurization is essential. Due to cost concerns, it is important to optimize the monitoring locations so as to use the least number of wells required to obtain the most comprehensive site characterization. The design of most monitoring networks is commonly based on a limited data set provided by existing measuring stations, which makes the optimization of the network a challenge.

There is an extensive literature on the general concept of environmental monitoring network optimization. Previous studies have focused on groundwater quality compliance monitoring (Loaiciga, 1989; Loaiciga et al., 1992; Meyer et al., 1994; Reed and Minsker, 2004), early warning systems for water distribution network contamination (Berry et al., 2006; Krause et al., 2008; Xu et al., 2010), contaminant source identification (Dhar and Datta, 2007; Mahar and Datta, 1997; Sun et al., 2006), and experiment-based frameworks for monitoring design (Bayer et al., 2010; Singh and Minsker, 2008; Ucinski and Patan, 2007). There have been very few studies focusing on the optimization of monitoring networks for the geologic storage of carbon dioxide. Most notable are the efforts of Yang et al. (2011), which evaluated detectability of $\mathrm{CO}_{2}$ leakage flux in the near surface environment for different monitoring network densities and parameter ranges; Sun et al. (2013), which proposed a new pressure-based monitoring network; Azzolina et al. (2014), which demonstrated a pressure-monitoring approach for improving the ability to detect smaller leaks in a more timely manner; and Wang and Small (2014), which 
characterized the $\mathrm{CO}_{2}$ leakage level in an idealized storage site through an assessment of the integrity of caprock inferred from injection zone pressure measurements. Pressure monitoring in the Above Zone Monitoring Interval, or AZMI, has been proposed for early detection of leakage (Hovorka et al., 2013) because of the fast traveling speed of pressure perturbations and the proximity of the AZMI to storage formations (Nordbotten et al., 2004). However, unlike the storage formations, the AZMI will be subjected to less pressure disturbance during injection activities, which will require a monitoring network capable of detecting these smaller signals as well as interpreting potential anomalous pressure signals. From an operations perspective, deep pressure monitoring wells are costly to drill and maintain-drilling and instrumentation costs can easily exceed $\$ 1$ million per well, which is in addition to annual maintenance and operation costs (U.S. Environmental Protection Agency, 2010). Thus, there is strong incentive to optimize the design of pressure-based monitoring networks (Sun et al., 2013).

In this paper, we focus on diffusive leakage of $\mathrm{CO}_{2}$ through the primary caprock and resulting pressure changes in the AZMI. The primary aim of this work is to characterize the time required to distinguish AZMI pressure outcomes and associated leakage scenarios through a probabilistic assessment of the integrity and permeability of the caprock and also the amount of $\mathrm{CO}_{2}$ injected into the reservoir. Four different fractured seal scenarios are designed for the primary caprock based on literature data. These scenarios represent caprock permeability from almost impermeable to highly permeable cases. We then model the probability distributions of pressure build-up in the AZMI for each of these scenarios, with the modeled pressure fields assumed to be observed with measurement errors. These distributions serve as the likelihood function for a Bayesian classification model, in which the posterior probabilities are computed for each of the four caprock fracture scenarios. We also evaluate the influence of the thickness of the caprock and the $\mathrm{CO}_{2}$ injection rate on the modeled pressure build-up and the subsequent performance of the Bayesian classification procedure. The modeling approach used in this study is based on the systematic framework shown in Figure 1.

\section{MODEL SETUP}

The $\mathrm{CO}_{2}$ storage system is modeled as a three-layer system with two aquifers separated by a 
sealing caprock of thickness $50 \mathrm{~m}$ (Figure 2 (a)). The lower aquifer is the storage reservoir where $\mathrm{CO}_{2}$ is injected at a base case rate of 1 MT per year for a period of 30 years. Higher and lower injection rates are also considered in a sensitivity analysis. The base case thickness of the reservoir is assumed to be $100 \mathrm{~m}$. The reservoir is located at a depth of $1000 \mathrm{~m}$. The areal extent of the subsurface storage system is defined as $10 \mathrm{~km} \times 10 \mathrm{~km}$. The assumed reservoir features are summarized in Table 1. Reservoir simulations (Figure 3) are conducted using TOUGH2. $\mathrm{CO}_{2}$ and brine flux from the seal are simulated for a period of 30 years of injection and 170 years of post-injection using NSealR. The above zone thickness used in this study is $50 \mathrm{~m}$. It is assumed that the AZMI layer has a porosity of 0.1 and a permeability of $10.5 \mathrm{mD}$. The residual $\mathrm{CO}_{2}$ and brine saturations were set at 0.01 and 0.02 respectively and the bubbling pressure was set to equal 0.01 MPa. Three base case observation wells screened at $900 \mathrm{~m}$ depth are also considered. The locations of the wells are shown in Figure 2 (b). The locations are chosen to be representative of a possible spatial layout, primarily for demonstration purposes.

The sealing caprock is modeled for four different fractured network scenarios: (I) fractured network with low aperture; (II) randomly distributed clusters of fractures with high apertures; (III) fractured network zone with high aperture near the injection well and; (IV) densely fractured network with high aperture. These four scenarios are assumed to be representative of the range of possible storage seal scenarios with an impermeable seal layer with almost no leakage possible represented by scenario (I); permeable and high risk storage scenarios represented by scenarios (III) and (IV) and a high integrity, almost impermeable seal with low leakage risk represented by scenario (II).

\subsection{FRACTURED SEAL SCENARIOS}

Quantitative assessment of storage system performance suggests that safe, effective long-term containment is highly probable in cases where there is an in-tact low-permeability seal to prevent vertical fluid migration. To date, however, little consideration has been given to scenarios in which the primary sealing layer contains regions fracturing or faulting that effectively represent heterogeneities in seal permeability. In this model, the sealing caprock is considered to be $50 \mathrm{~m}$ thick. In order to add heterogeneity to our analysis, we simulate semi-stochastic fracture network 
characteristics using FRACGEN (McKoy et al., 2006). This software is specifically designed for modeling fracture networks and fractured reservoirs. The fracture network used in this model is based on the stochastic allocation of fracture lengths, positions, orientations and density in space. Model details are provided in the Supporting Information.

For demonstration purpose, four representative heterogeneous fractured seal types are defined and used for the model analysis as represented graphically in Figure 4. Fracture modeling details used in each of these scenarios can be found in Table $S$-2 of the Supporting Information. The four seal scenarios generated for this work are expected to be representative of fractured seal scenarios ranging from an almost impermeable caprock layer (good storage seal case) to a highly permeable caprock layer (worst storage seal case). These scenarios are intended to illustrate how alternative seal fracture properties can be defined, simulated, and used to induce a leakage and fluid migration pattern from the injection zone, through the caprock and into the AZMI. Since the NSealR model uses seal permeability as an input, we use a parallel plate model for fractures to compute the effective permeability of the fractured networks.

\subsection{EFFECTIVE CAPROCK PERMEABILITY}

Defining the aperture and the permeability of a fracture separately seems counterintuitive, since these parameters are considered to be mutually dependent, and directly related if the fractures are rectangular slits. Parallel plate theory (Snow, 1964; Sarkar, 2004) is used to compute fracture permeability (See Supporting Information). Figure 5 shows the effective permeability plot for the four fractured scenarios. The calculated permeabilities are then used to compute flow through the seal using NSealR. As this study focuses on the above zone pressure build up due to associated diffusive $\mathrm{CO}_{2}$ leakage through the primary caprock, we determine whether the four different fractured caprock scenarios lead to pressure build up profiles that are statistically distinguishable (Table 2).

\subsection{AZMI ROM RESULTS}

The AZMI ROM (Namhata et al., 2016) is used to calculate the above zone pressure build up for 
each of the caprock scenarios. Figure 6 presents the changes in pressure responses in the AZMI over time, generated using the flux from the seal for each of the fracture scenarios for the simulation periods previously identified.

\section{BAYESIAN CLASSIFICATION METHODOLOGY}

An expert's belief regarding the relative probability that each caprock fracture scenario is present at a site can be combined with observed pressure monitoring and modeling results using Bayesian classification theory. The belief of the expert is assumed to be a prior distribution of the presence of each of the four scenarios at a $\mathrm{CO}_{2}$ storage site. In the Bayesian classification methodology, the posterior distribution is then derived by combining the prior distribution with the monitored pressure at the three monitoring locations. If there is no information on the prior distribution, an equally probable prior is assumed so that the results will totally depend on the monitored (or, modeled) pressure outputs. In this case, the posterior probability of a fracture scenario is proportional to the likelihood function for the modeled pressure outputs (time and location dependent), given each fracture scenario.

To characterize the performance of the classification procedure, simulated leakage - pressure outcomes are generated for each scenario and translated into an assumed sequence of AZMI pressure measurements. The likelihood function for the above zone pressure measurements using the AZMI ROM has two components: firstly, the uncertainty in the true value of the above zone pressure that results from uncertainties in caprock fracture properties; and secondly, the uncertainty that might be associated with the modeling error. The first uncertainty is captured by 100 discrete FRACGEN simulation results. The latter is captured by assuming log normal measurement error function that maps simulated modeling results to pressure values that are assumed to be measured. To determine the ability to infer true caprock fracture scenario type at the site, we simulate multiple realizations for each fracture scenario, assume they are measured with error, and use the Bayesian classification procedure to infer the probability that each scenario is present. Good performance occurs when the procedure predicts high probability for the scenario used to simulate the leakage and associated pressure realizations (and low probability for the others). 


\subsection{Mean Pressure Buildup}

As shown in Figure 2 (b), we have assigned three above zone monitoring wells for our base case analysis. The number and location of the monitoring wells chosen in this study are illustrative. The regions closer to the injection well are expected to see higher pressure build up, making them an obvious choice for monitoring. The three locations chosen are in regions right above or near the injection well. In this analysis, we calculate the above zone pressure build up due to $\mathrm{CO}_{2}$ injection in the storage reservoir for each of the fractured seal scenarios (Figure 6). The pressure build ups at the three monitoring locations are then calculated for each scenarios and at each time step. The mean of the monitored pressure build up is then used for the analysis. The purpose of choosing the mean pressure build up $\left(\Delta P_{\text {simulated }}\right)$ over individual monitoring point analysis is that it provides the ability of the mean pressure build up to capture the spatial variability in output predictions over individual analysis. The mean pressure build up range from the 100 simulations for each scenario is shown in Figure 7.

\subsection{Inferring Fracture Scenario}

Assuming no knowledge about the seal, we assign equal prior probability $(=0.25)$ for each of the four fracture scenarios (Table 2). The mean above zone pressure build up in the three monitoring wells $\left(\Delta P_{\text {simulated }}\right)$ is chosen to be the variable for analysis of the fracture seal scenarios. The expected effective permeabilities of the caprock for each of the fracture scenarios are shown in Table 2. The greater the effective permeability of the seal, the more will be the pressure build up in the AZMI resulting in higher $\Delta P_{\text {simulated }}$. For the cases considered and simulated in our study, the $\Delta P_{\text {simulated }}$ ranges from 0 to $0.325 \mathrm{MPa}$. Since we consider measurement errors, the observed values tend to extend beyond this range. For simplicity in the statistical analysis (mainly for lognormal analysis), we add a minimum threshold value of $0.001 \mathrm{MPa}$ to the $\Delta P_{\text {simulated. }}$.

We then estimate the likelihood of observing $\Delta P_{\text {simulated }}$ given each caprock fracture scenario, $f\left(\Delta P_{\text {simulated }} \mid\right.$ Scenario $\left.j\right)$, where $j$ is the scenario number. We first generate 100 realizations of each caprock fracture scenario incorporating values shown in Table 1 , and compute $\Delta P_{\text {simulated. }}$ 
Secondly, we simulate the effects of measurement errors, by assuming that the above zone pressure build ups $\left(\Delta P_{\text {measured }}\right)$ are log-normally distributed about the model simulation values:

$\Delta P_{\text {measured }} \sim \operatorname{lognormal}(a, b)$

where,

$$
\begin{aligned}
& a=\log \left(\Delta P_{\text {simulated }}\right) \\
& b=\left[\log \left(c . v^{2}+1\right)\right]^{1 / 2}
\end{aligned}
$$

We calculate the median $\Delta P_{\text {simulated }}$ values from each FRACGEN simulation for the respective lognormal distributions of $\Delta P_{\text {measured }}$ (specifying the parameters $a$ representing the logarithm of the median of the respective measurements). The second parameter $b$ represents the standard deviation of the logarithm of $\Delta P_{\text {measured }}$. These second parameters are specified by the coefficients of variation (c.v.) of $\Delta P_{\text {measured }}$.

Thus, the lognormal distribution of $\Delta P_{\text {measured }}$ is computed using the simulation results of $\Delta P_{\text {simulated }} i$ for each simulation $i$ and at each time step $t$ and the assumed measurement error (coefficient of variation here) for each $\Delta P_{\text {simulated }}$ measurement (determined by $b$ ). The pdf of the lognormal distribution serves as the likelihood function for the pressure observations given the simulation result:

$$
f\left(\Delta P_{\text {measured }}(t) \mid a_{i}, b\right)=\log \left(\frac{1}{\Delta P_{\text {measured }}(t) \times a_{i} \times \sqrt{2 \pi}} \exp \left\{\frac{-\left(\log \left(\Delta P_{\text {measured }}(t)\right)-a_{i}\right)^{2}}{2 b^{2}}\right\}\right)
$$

The overall log-likelihood of a given fracture scenario is given by the sum of individual loglikelihoods from all 100 simulations for each case:

$\log$-likelihood $(\operatorname{scenario} j(t))=\sum_{i=1}^{100} f\left(\Delta P_{\text {measured }}(t) \mid a_{i}, b\right)$

Using Bayes theorem, the prior distribution of each caprock fracture scenario and the likelihood 
function are combined to calculate the posterior distribution of each caprock fracture scenario given by:

$$
\pi\left(\text { scenario } j(t) \mid \Delta \mathrm{P}_{\text {measured }}(t)\right)=\frac{\exp \left[f\left(\Delta \mathrm{P}_{\text {measured }}(t) \mid \text { scenario } j\right)\right] \times \operatorname{Prob}[\text { scenario } j]}{\sum_{i=1}^{4} \exp \left[f\left(\Delta \mathrm{P}_{\text {measured }}(t) \mid \text { scenario } i\right)\right] \times \operatorname{Prob}[\text { scenario } i]}
$$

\subsection{Time to Detect Leakage}

The primary aim of this work is to compute the time required to distinguish above zone pressure outcomes and the associated leakage scenarios. The time to detect leakage or no leakage are calculated using the posterior probabilities given each of the four scenarios. We compute two different time values, (a) the time to no leakage assurance given there is no leakage $\left(T_{\text {no leak }}\right)$ and, (b) time to leakage confirmation given there is a leakage $\left(T_{\text {leak }}\right)$. For simplicity we assume that the fracture scenario $\mathrm{I}$ is a no leakage scenario. $T_{\text {no leak }}$ provides an assessment of the system behavior by calculating the time required to correctly conclude that there is leakage from the caprock assuming that the caprock is of scenario I type i.e., almost impermeable caprock. $T_{\text {leak }}$ provides an estimate of how long it takes to correctly conclude that there is leakage from the caprock assuming that the caprock is of scenario types II, III or IV. Posterior probability thresholds of $90 \%$ are assumed to be required for drawing the no-leak or leak scenario inferences. Mathematically,

$T_{\text {no leak }}=t$ when $\pi\left(\right.$ scenario $\left.\mathrm{I}(t) \mid \Delta \mathrm{P}_{\text {measured }}(t)\right) \geq 0.9 \mid$ scenario $\left.\mathrm{I}(t)\right)$

and,

$T_{\text {leak }}=t$ when $\pi\left(\right.$ scenario $\left.\mathrm{I}(t) \mid \Delta \mathrm{P}_{\text {measured }}(t)\right)<0.9 \mid$ scenario $\left.j(t)\right): j \neq 1$

\subsection{Influence of Input Parameters}

Along with the uncertainties in fracture properties (represented in Table S-2, See Supporting Information), there can be other uncertainties associated with $\mathrm{CO}_{2}$ storage system that impact 
the above zone pressure monitoring results. To illustrate the sensitivity of model predictions to variations in selected modeling parameters, we change the $\mathrm{CO}_{2}$ injection rate and thickness of the primary caprock to compute their effect on simulation results and the probability of properly inferring caprock fracture scenarios. The base case model was set up for a caprock of thickness $50 \mathrm{~m}$ and an injection rate of $1 \mathrm{MT} /$ year for 30 years. We make changes to the base case and also present simulation results for a $10 \mathrm{~m}$ and $100 \mathrm{~m}$ thick caprock; and injection rates of $10 \mathrm{MT} /$ year and $50 \mathrm{MT} /$ year.

\section{RESULTS}

The base case model was implemented for each of the four caprock fracture scenarios. The above zone pressure buildup was then calculated using the AZMI ROM. For posterior analysis we use the mean pressure buildup from three monitoring well locations as described previously. Figure 8 shows the posterior probability of each of the caprock fracture scenarios as a function of time from the start of injection. The posterior probabilities are calculated for each of the fracture scenarios given the simulation results from a particular fracture scenario. This demonstrates whether and how quickly a true fracture scenario can be distinguished from the others considered, based solely on pressure measurements in the AZMI. Since the fracture scenarios are general in nature, chosen to be representative of probable caprock types with different leakage potential, the results are intended to provide a simple representation of how the monitored pressure will evolve and be interpreted for similar scenarios.

In Figure 8, we present the posterior distribution of each scenario given one of the four scenarios. All of the plots have been shown until 35 years from the start of injection. Since the main aim of this work is to identify the time required to distinguish above zone pressure outcomes and the associated leakage scenarios, we concluded from all the simulations that the maximum associated time is less than 35 years. In each of the plots, there is no change in posterior probability from the prior values $(=0.25)$ in the first 3 years. It can be seen from the scenario I plot that the posterior probability of scenario I given scenario I reaches 0.90 at 5 years from the start of injection. This is expected because with more and more monitoring, the posterior probability is expected to increase for a particular scenario given the same scenario. A similar 
trend is expected for the other scenarios, but they take longer to reach a statistically significant posterior probability $(=0.90)$. While the leakage scenarios can, as a group, be distinguished from the non-leakage case, the method is often unable to distinguish the scenarios from each other. This is because there is no flow of $\mathrm{CO}_{2}$ in the initial years to the AZMI, resulting in no pressure buildup. Scenario I being distinctively different from the rest of the scenarios has a very distinct posterior pattern. Since this scenario represents a negligible leakage case, we don't see the posterior of other scenarios, especially scenarios III and IV, increasing. Scenario II being a low leakage case, there will be no/ very less migration of fluids, hence there is a slight increase in its posterior initially.

For posterior probability plots of scenarios III and IV given scenarios III and IV respectively, there is an increasing trend as these scenarios are representative of high leakage cases, with almost no leakage effect from scenario I and very low impact from scenario II. Given scenario III, we see an increase in the posterior probability of scenarios II and IV at a later stage of injection since both these scenarios also have increasing pressure buildup over time. But the posteriors remain less compared to the posterior of scenario III. In case of scenario II, which is representative of low permeability caprock with the potential for a low to moderate amount of $\mathrm{CO}_{2}$ leakage, we see higher probability of scenario I compared to scenario II itself in the first 5 years. The reason being in its initial years, there is negligible leakage of $\mathrm{CO}_{2}$ in scenario II, similar to that of scenario I. So there is a clear impact on posteriors for that case.

We next conducted sensitivity analysis to determine the effect of changing caprock thickness (from a base case of $50 \mathrm{~m}$ ) and the injection rate (from a base case of $1 \mathrm{MT} /$ year) on model inferences to:

(a) Caprock thickness of $10 \mathrm{~m}$ and $100 \mathrm{~m}$

(b) Injection rate of $0.25 \mathrm{MT} /$ year and $5 \mathrm{MT} /$ year.

Figures 9 and 10 shows the inferred posterior probabilities over time for caprock thickness $10 \mathrm{~m}$ and $100 \mathrm{~m}$, respectively, keeping all the other inputs to the simulation the same as the base case 
simulation. Figures 11 and 12 shows the posterior distribution over time for injection rate of 0.25 MT/ year and 5 MT/ year, respectively, for 30 years. From Figures 9 and 10 it can be seen that the general characteristic trend of the posteriors are similar to that in Figure 8. With a decrease in caprock thickness to $10 \mathrm{~m}$ (Figure 9), there will be more leakage of $\mathrm{CO}_{2}$ in the AZMI compared to that from a caprock of thickness $50 \mathrm{~m}$. This distinctive feature is captured for posteriors given scenario II plot, where the time to predict confidently that the change in pressure is due to scenario II is increased from the base case. For scenario III and IV, which are in general high leakage scenarios, the pattern is similar to that of the base case. When the caprock thickness is increased to $100 \mathrm{~m}$ (Figure 10), we expect lower $\mathrm{CO}_{2}$ leakage into the AZMI compared to that of the base case. In this case too, scenario II cannot be distinguished from scenario I as quickly as the rest of the scenarios. Comparing the curves in Figures 9 and 10 to that of 11 suggests that changing the caprock thickness has very little effect on the magnitude of predicted probabilities. This is not the case when we change the injection rates. A lowering of the injection rates yields a lower or similar statistical ability to infer the caprock fracture scenario compared to that of the base case. Increasing the injection rate (Figure 11) yields an increase in the statistical ability for inferring the caprock fracture scenario. This is as expected - increasing the injection rate increases the likelihood of $\mathrm{CO}_{2}$ leakage and in turn results in an increase in above zone pressures yielding a more likely detection of caprock fracture scenarios. The present model is formulated based on the assumption that there is no prior scenario-specific knowledge. In case of prior information about the caprock geology, the probability of inferring the caprock scenarios will also change. In this study, to show the impact of prior knowledge on caprock type inference and also the time taken to detect a leak/ no leak scenario, we slightly change the prior information situation from that of Table 2. The prior of Scenario I is changed to 0.50 and the rest of the scenarios are taken to have a 0.1667 prior probability. The resulting inferred posterior probabilities are shown in Figure 13. It can be seen that, with higher prior information of Scenario I, the posterior probability of Scenario I given Scenario I is quite high from the beginning. In the Scenario II plot it can be seen that the time to infer the leakage due to Scenario II has changed a lot. The other scenarios have almost similar inference ability to that of the base case.

As the primary aim of this work is to calculate $T_{\text {no leak }}$ and $T_{\text {leak }}$, we present the respective times 
for the base case and the four other scenarios in Tables 3 and 4. In Table 3, the time to no leak assurance is shown. This will help in understanding the minimum time required to confidently say that there are no significant monitorable pressure changes in the AZMI. The higher the caprock thickness and the lower the injection rate, more will be the time required to reach a no leak assurance conclusion since we expect to have low leakage in such situations. For an opposite scenario, with low caprock thickness and high injection rate, the time to no leak assurance will be much less. In Table 4, the time to leakage confirmation is shown. This is the time needed to conclude that most probably there is a leakage in the system based on the pressure data. The most time needed to conclude that there is a leakage in the system occurs with scenario II when the caprock thickness is low and the injection rate is low and medium. This occurs because, for such a scenario, there is expected to be much less leakage or no leakage. In order to reach a statistically significant conclusion that there is a chance of leakage, a long time is required. On the other hand, in scenario III where the fractured zones are above the injection well and below the monitoring wells, we expect to see high leakage rates for almost all of the scenarios. Thus the time to leakage confirmation is much lower in this case. For scenario IV, where the caprock is highly fractured throughout the space, we expect a higher distribution of flux and in turn higher above zone pressure. Thus the ability to detect a leak is also lower for this scenario. This can be seen from Table 4, where the average time to detect a leak is 6 years.

\section{CONCLUSION}

In this study, we simulate a caprock with semi-stochastic fracture network characteristics using FRACGEN (McKoy et al., 2006). For demonstration purpose, four representative heterogeneous fractured seal types are generated, ranging from an almost impermeable caprock layer (good storage seal case) to a highly permeable caprock layer (worst storage seal case). Existing reduced order models (ROMs) are used to predict the pressure response in the Above Zone Monitoring Interval (AZMI) and flux response above the caprock for a hypothetical base case $\mathrm{CO}_{2}$ storage scenario. The probability distributions of pressure build-up in the AZMI are modeled for each of the four caprock fracture scenarios. The modeled pressure fields are assumed to be observed with measurement errors. A Bayesian classification methodology is then developed where the pressure distributions are used as likelihood functions to compute posterior probabilities for each 
scenario. The Bayesian model is primarily used to calculate two parameters: firstly the inferred probability of a given fracture scenario and secondly, the time required to distinguish above zone pressure outcomes and the associated leakage scenarios. The results indicate that with an ideal storage case where the caprock is very thick and almost impermeable, the time taken to infer that no leak is occuring is relatively short. Similarly, if the storage scenario is not ideal for $\mathrm{CO}_{2}$ injection, i.e., the thickness of the seal is low and it is highly fractured with high permeability, then the time to infer that leakage is occurring is short. The injection rate and the thickness of the caprock have influence on the predicted caprock fracture scenario and the detection power of the simulated above zone pressure monitoring. Reduction in uncertainties of caprock geology, especially more knowledge of fracture network properties through site characterization, can lead to higher confidence in the predicted caprock fracture scenario and also improve the statistical power of detecting leakage through the caprock. Above zone pressure monitoring, combined with other monitoring techniques, such as groundwater quality monitoring, seismic monitoring, surface deformation monitoring and any other applicable monitoring techniques can be used to predict $\mathrm{CO}_{2}$ leakage rates from the reservoir with a higher confidence. 


\section{DISCLAIMER}

This report was prepared as an account of work sponsored by an agency of the United States Government. Neither the United States Government nor any agency thereof, nor any of their employees, makes any warranty, express or implied, or assumes any legal liability or responsibility for the accuracy, completeness, or usefulness of any information, apparatus, product, or process disclosed, or represents that its use would not infringe privately owned rights. Reference therein to any specific commercial product, process, or service by trade name, trademark, manufacturer, or otherwise does not necessarily constitute or imply its endorsement, recommendation, or favoring by the United States Government or any agency thereof. The views and opinions of authors expressed therein do not necessarily state or reflect those of the United States Government or any agency thereof.

\section{ACKNOWLEDGMENTS}

This work was completed as part of the National Risk Assessment Partnership (NRAP) project. Support for this project came from the U.S. Department of Energy's (DOE) Office of Fossil Energy's CCS and Crosscutting Research Programs, by the Department of Civil and Environmental Engineering and the Bertucci fellowship program at Carnegie Mellon University, and by training fellowship through the Oak Ridge Institute for Science \& Education (ORISE). The authors would like to thank Grant Bromhal, Mark L. McKoy, Neal W. Sams, Evgeniy M. Myshakin, Traci Rodosta, Robert Romanosky, M. Kylee Rice, and Steven Seachman of NETL and Mark Ackwiecz and Regis Conrad of U.S. DOE, Fossil Energy for their technical direction and Programmatic guidance; Liwei Zhang, Zan Wang and Ya-Mei Yang of NETL/ ORISE at NETL; and Seth King and Ernest Lindner of AECOM, Inc. at NETL for their valuable technical comments. 


\section{REFERENCES}

Azzolina, N. A., Small, M. J., Nakles, D. V., \& Bromhal, G. S. (2014). Effectiveness of subsurface pressure monitoring for brine leakage detection in an uncertain $\mathrm{CO}_{2}$ sequestration system. Stochastic Environmental Research and Risk Assessment, 28(4), 895-909.

Bayer, P., de Paly, M., Bürger, C.M. (2010) Optimization of high-reliability-based hydrological design problems by robust automatic sampling of critical model realizations. Water Resour. Res. 46, W05504.

Birkholzer, J.T.; Zhou, Q. (2009) Basin-scale hydrogeologic impacts of $\mathrm{CO}_{2}$ storage: capacity and regulatory implications. Int. J. Greenh. Gas Con. 3, 745-756.

Dhar, A., Datta, B. (2007) Multi objective design of dynamic monitoring networks for detection of groundwater pollution. J. Water Resour. Plan. Manag. 133, 329-338.

Hovorka, S.D., Meckel, T.A., Trevĩno, R.H., (2013) Monitoring a large-volume injection at Cranfield, Mississippi-project design and recommendations. Int. J. Greenhouse Gas Control.

IPCC (Intergovernmental panel on climate change) (2005) IPCC Special Report on Carbon Dioxide Capture and Storage. Prepared by Working Group III of the Intergovernmental Panel on Climate Change [Metz, B., O. Davidson, H. C. de Coninck, M. Loos, and L. A. Meyer (eds.)]. Cambridge University Press, Cambridge, United Kingdom and New York, NY, USA, 442 pp.

Jung, Y.; Zhou, Q.; Birkholzer, J.T. (2013). Early detection of brine and $\mathrm{CO}_{2}$ leakage through abandoned wells using pressure and surface-deformation monitoring data: concept and demonstration. Adv. Water Resources.

Loaiciga, H.A. (1989) An optimization approach for groundwater quality monitoring network design. Water Resour. Res. 25, 1771-1782.

Loaiciga, H.A.; Charbeneau, R.J.; Everett, L.G.; Fogg, G.E.; Hobbs, B.F.; Rouhani, S. (1992) 
Review of ground-water quality monitoring network design. J. Hydraul. Eng. 118,1137.

Mahar, P.S., Datta, B. (1997) Optimal monitoring network and ground-water-pollution source identification. J. Water Resour. Plan. Manag. 123, 199-207.

McKoy et al. (2006) FRACGEN User's Guide; U.S. Department of Energy, National Energy Technology Laboratory: Morgantown, WV.

Meyer, P.D.; Valocchi, A.J.; Eheart, J.W. (1994) Monitoring network design to provide initial detection of groundwater contamination. Water Resour. Res. 30, 2647-2659.

Namhata, A.; Zhang, L.; Dilmore, R.M.; Oladyshkin, S.; Nakles, D.V. (2016) Modeling pressure changes due to migration of fluids into the Above Zone Monitoring Interval of a Geologic Carbon Storage Site, International Journal of Greenhouse Gas Control, Submitted.

Nordbotten J, Celia M, Bachu S (2004) Analytical solutions for leakage rates through abandoned wells. Water Resources Research, 40

Pruess, K., (2004). Numerical simulation of $\mathrm{CO}_{2}$ leakage from a geologic disposal reservoir, including transitions from super-to subcritical conditions, and boiling of liquid $\mathrm{CO}_{2}$. SPE Journal, 9(02), 237-248.

Reed, P.M.; Minsker, B.S. (2004) Striking the balance: long-term groundwater monitoring design for conflicting objectives. J. Water Resour. Plan. Manag. 130, 140-149.

Sarkar, S., Toksöz, M. N., Burns, D.R. (2004), Fluid Flow Modeling in Fractures, Dept. of Earth, Atmospheric and Planetary Sciences, Massachusetts Institute of Technology, Cambridge, MA.

Singh, A., Minsker, B.S. (2008) Uncertainty-based multi objective optimization of groundwater remediation design. Water Resour. Res. 44, W02404.

Snow, D.T. (1964), A parallel plate model of fractured permeable media, Ph.D. Thesis, University of California, Berkeley, USA.

Sun, A.Y., Painter, S.L., Wittmeyer, G.W. (2006) A robust approach for iterative contaminant source location and release history recovery. J. Contam. Hydrol. 88,181-196.

Sun, A.Y.; Nicot, J.P. (2012) Inversion of pressure anomaly data for detecting leakage at geologic carbon sequestration sites. Adv. Water Resour., 20-29.

Sun, A.Y., Nicot, J.-P., Zhang, X. (2013) Optimal design of pressure-based leakage detection 
monitoring networks for geologic carbon sequestration repositories. Int. J. Greenh. Gas Control 19, 251-261.

Ucinski, D., Patan, M. (2007) D-optimal design of a monitoring network for parameter estimation of distributed systems. J. Global Optimiz. 39, 291-322.

U.S. Environmental Protection Agency, (2010) Geologic $\mathrm{CO}_{2}$ Sequestration Technology and Cost Analysis. EPA, Washington, DC.

Wang, Z.; Small, M.J. (2014) A Bayesian approach to $\mathrm{CO}_{2}$ leakage detection at saline sequestration sites using pressure measurements. International Journal of Greenhouse Gas Control. 30, 188-196.

Yang, Y.M., Small, M.J., Ogretim, E.O., Gray, D.D., Bromhal, G.S., Strazisar, B.R., Wells, A.W. (2011) Probabilistic design of a near-surface $\mathrm{CO}_{2}$ leak detection system. Environ. Sci. Technol. 45, 6380-6387. 


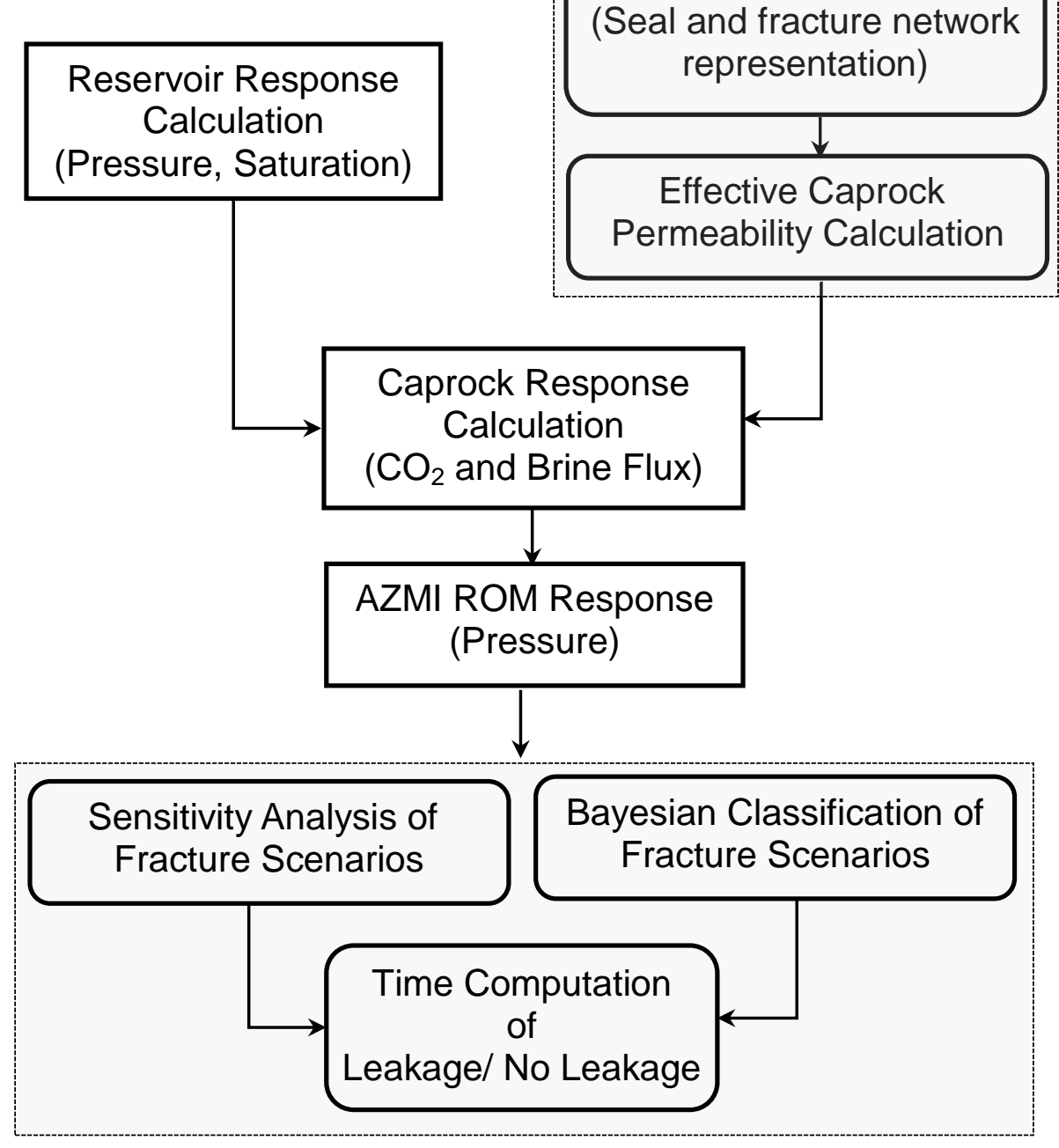

Figure 1: Schematic framework of Bayesian design for above zone pressure monitoring. 


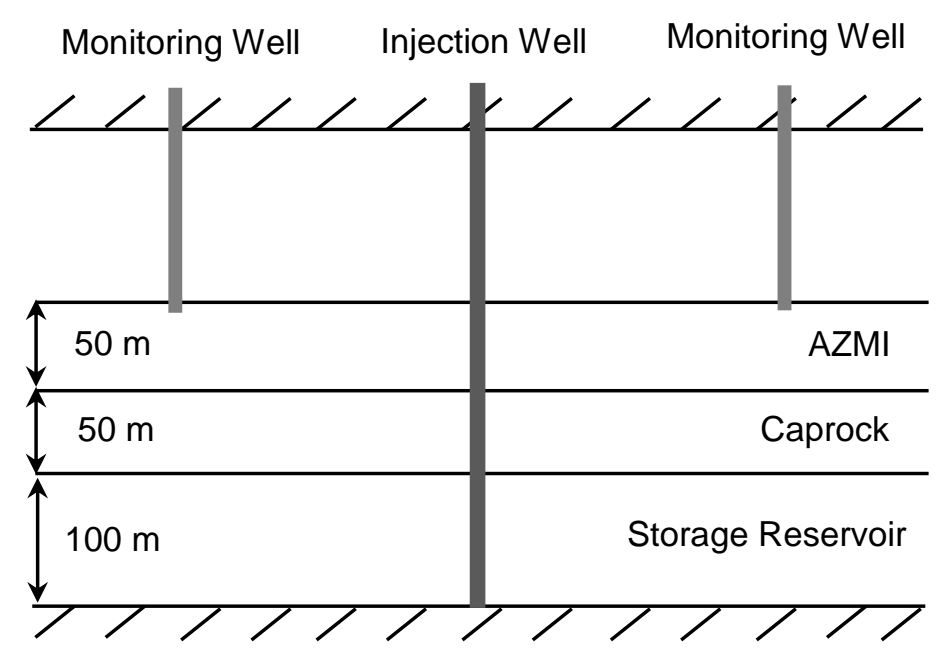

(a)

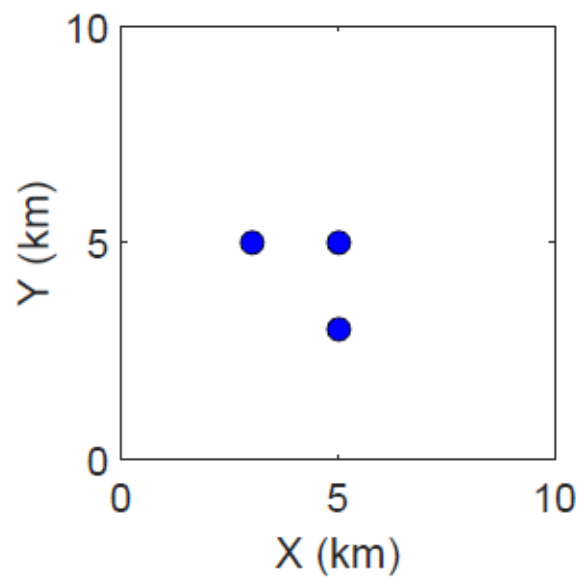

(b)

Figure 2: (a) Schematic diagram of the simplified geological model used for the base case study; (b) Top view of the spatial locations of base case monitoring wells. 

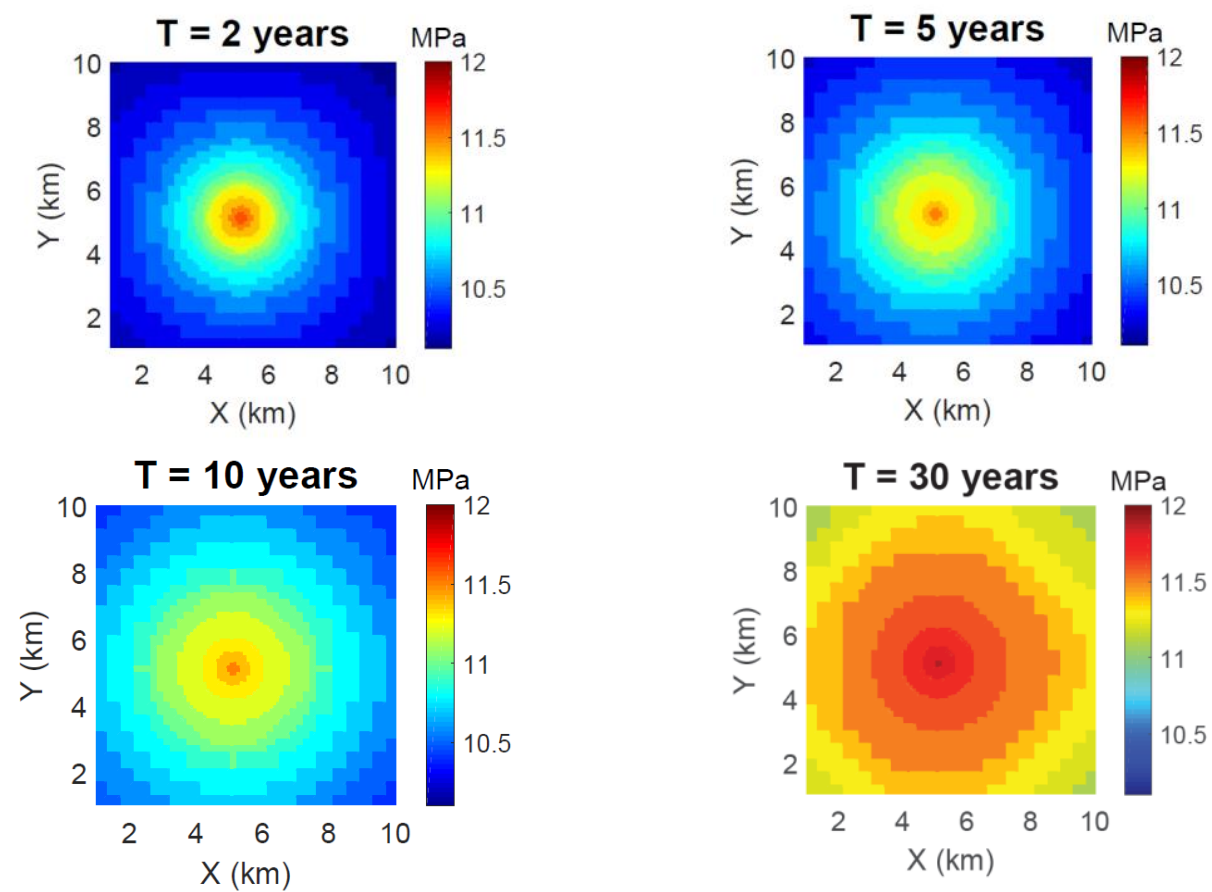

(a)
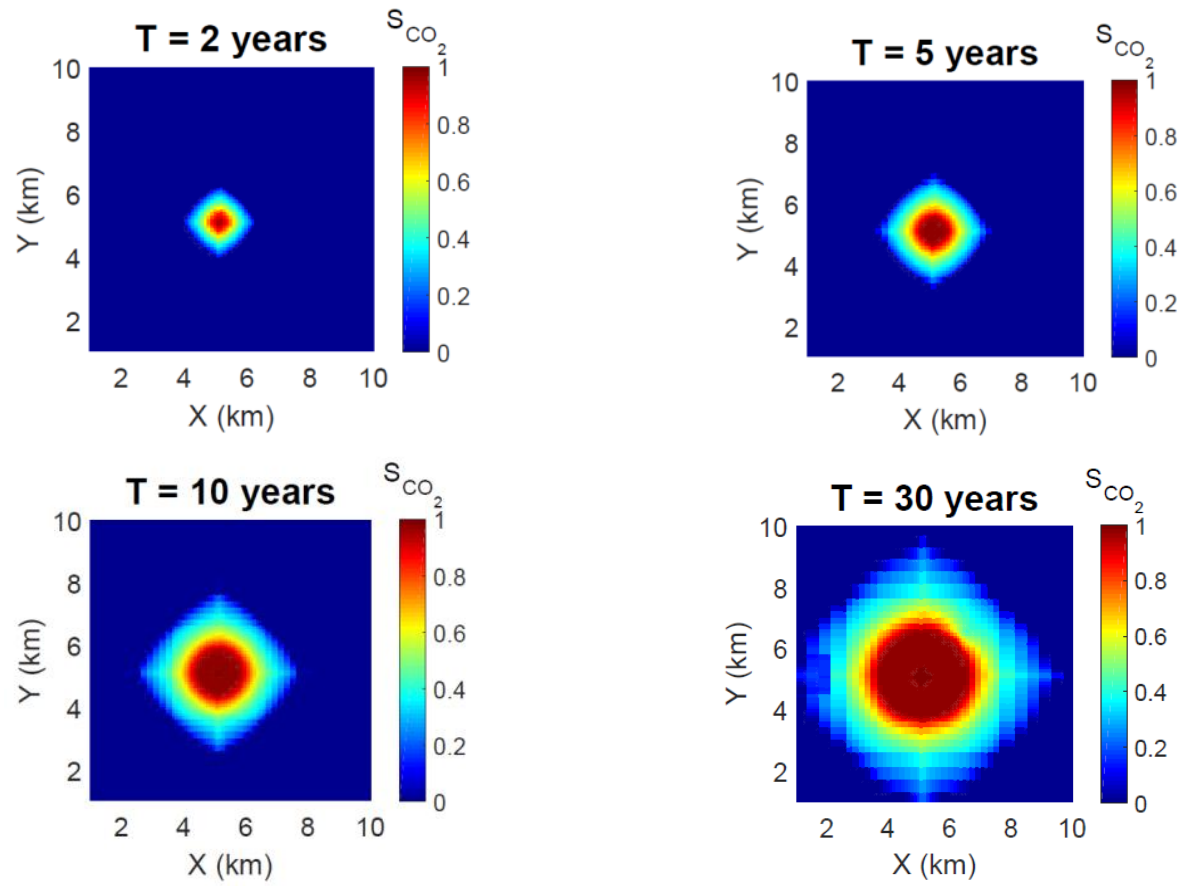

(b)

Figure 3: Evolution of (a) pressure (in $\mathrm{MPa}$ ) and, (b) $\mathrm{CO}_{2}$ saturation at the top of the injection reservoir at 2,5, 10 and 30 years after the start of $\mathrm{CO}_{2}$ injection. 


\section{Scenarios}
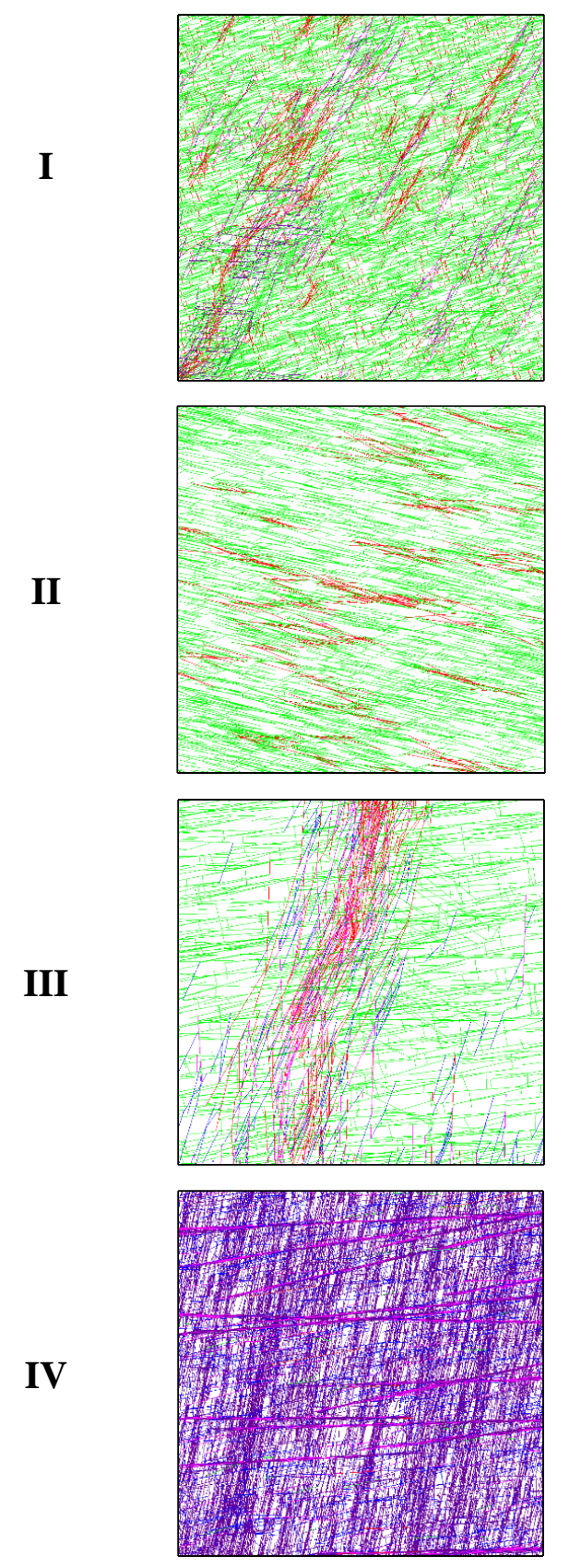

Fracture apertures (in ft.)
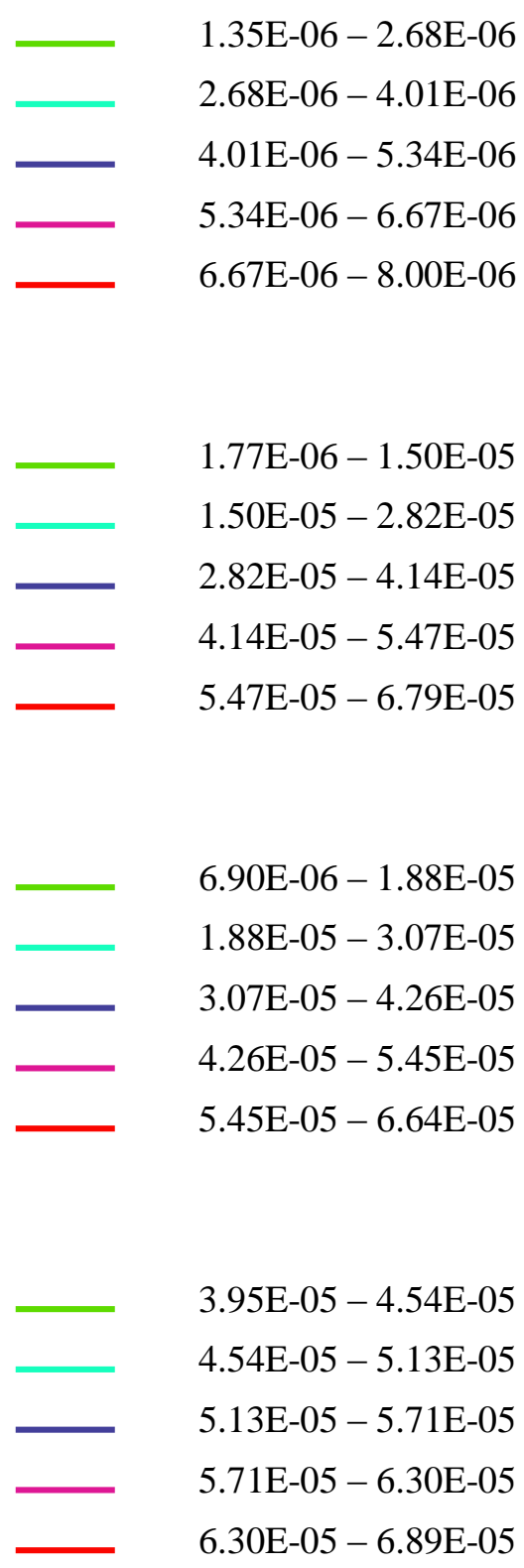

Figure 4: Graphical representation of fractured seal scenarios: (I) fractured network with low aperture; (II) densely fractured network with high aperture; (III) randomly distributed clusters of fractures with high apertures and; (IV) fractured network zone with high aperture above injection well. 


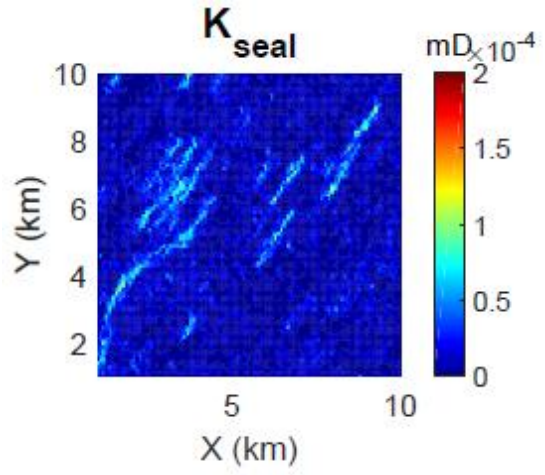

(a)

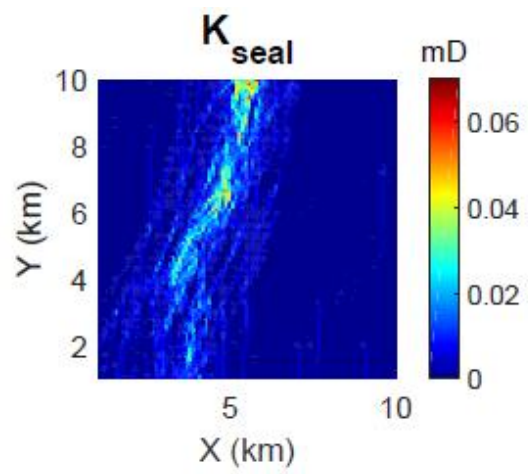

(c)

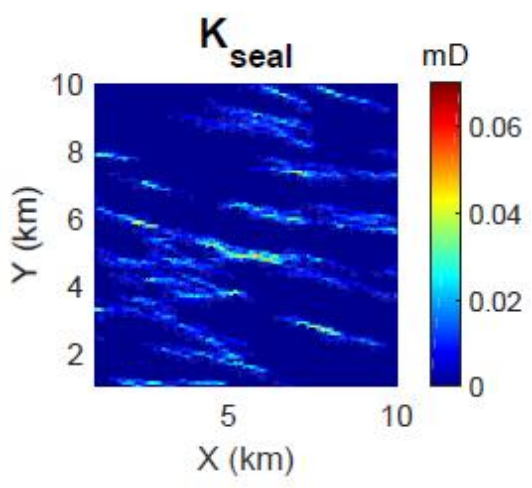

(b)

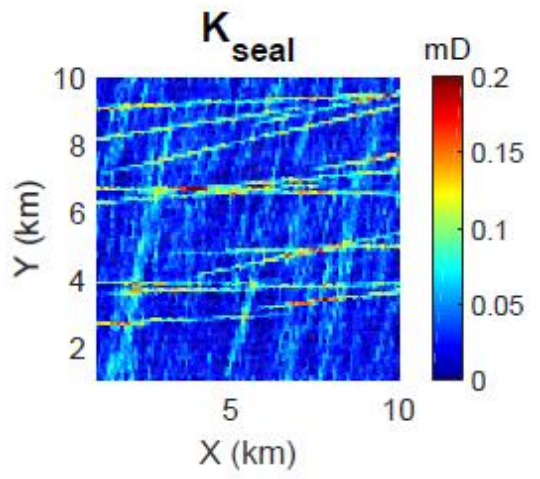

(d)

Figure 5: Calculated effective caprock permeability (in $\mathrm{mD}$ ) for base case model with four fracture scenarios: (a) Scenario I, (b) Scenario II, (c) Scenario III and, (d) Scenario IV. 

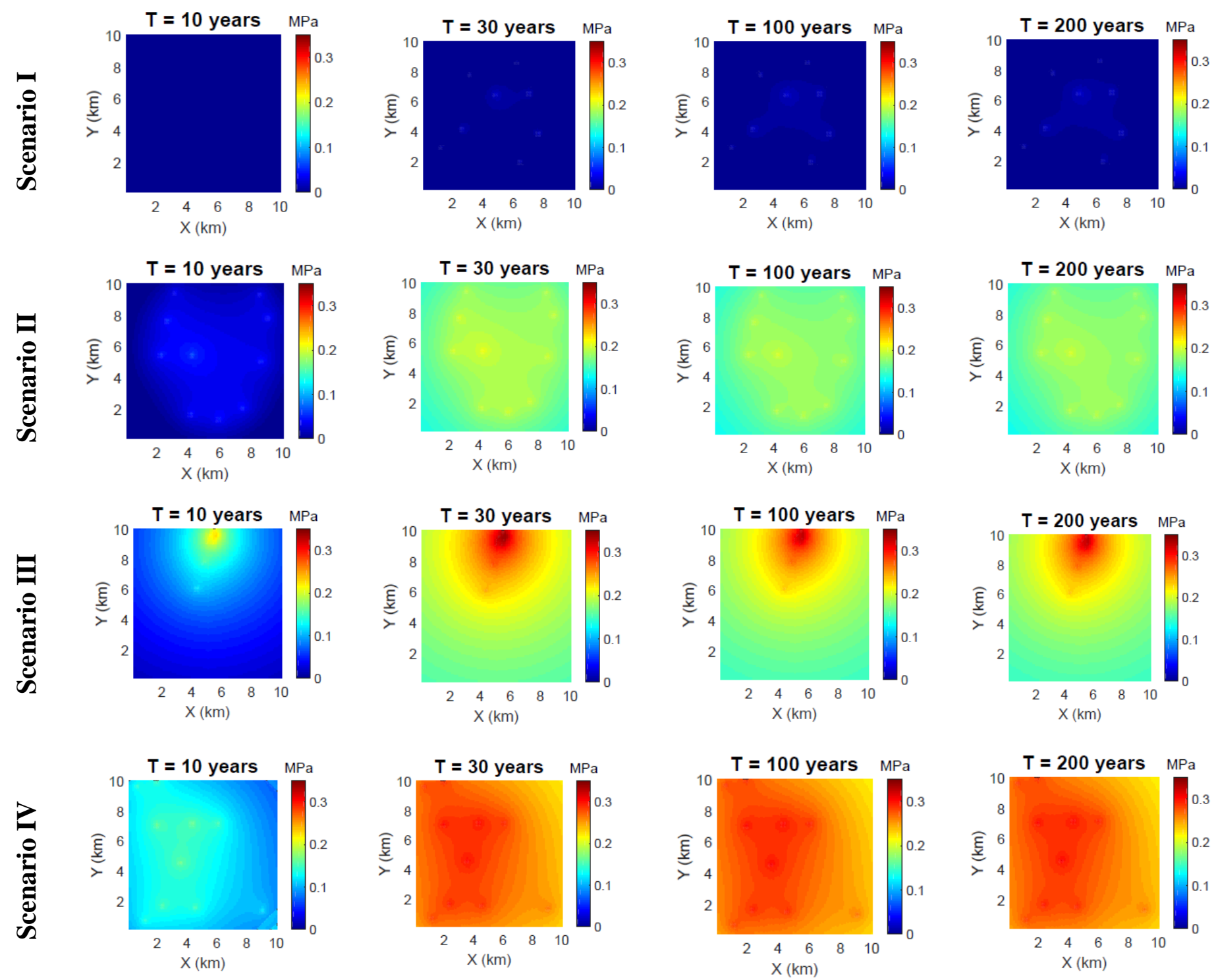

Figure 6: Change in pressure response (in MPa) at the top of AZMI through 200 years from the start of injection for the base case model with four caprock fracture scenarios. 

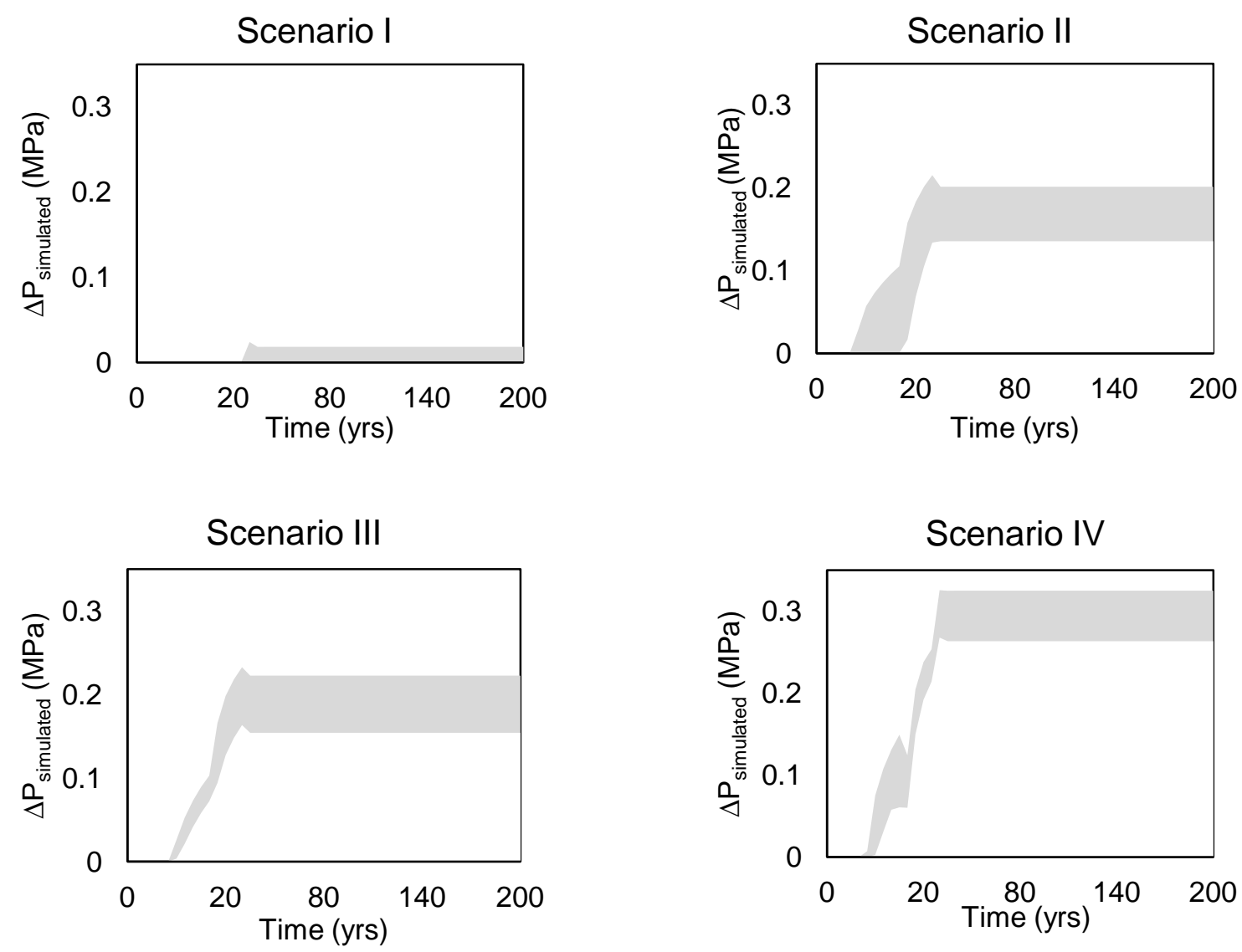

Figure 7: Range of mean of pressure build up at three designated monitoring well locations for all caprock fracture scenarios over time. 

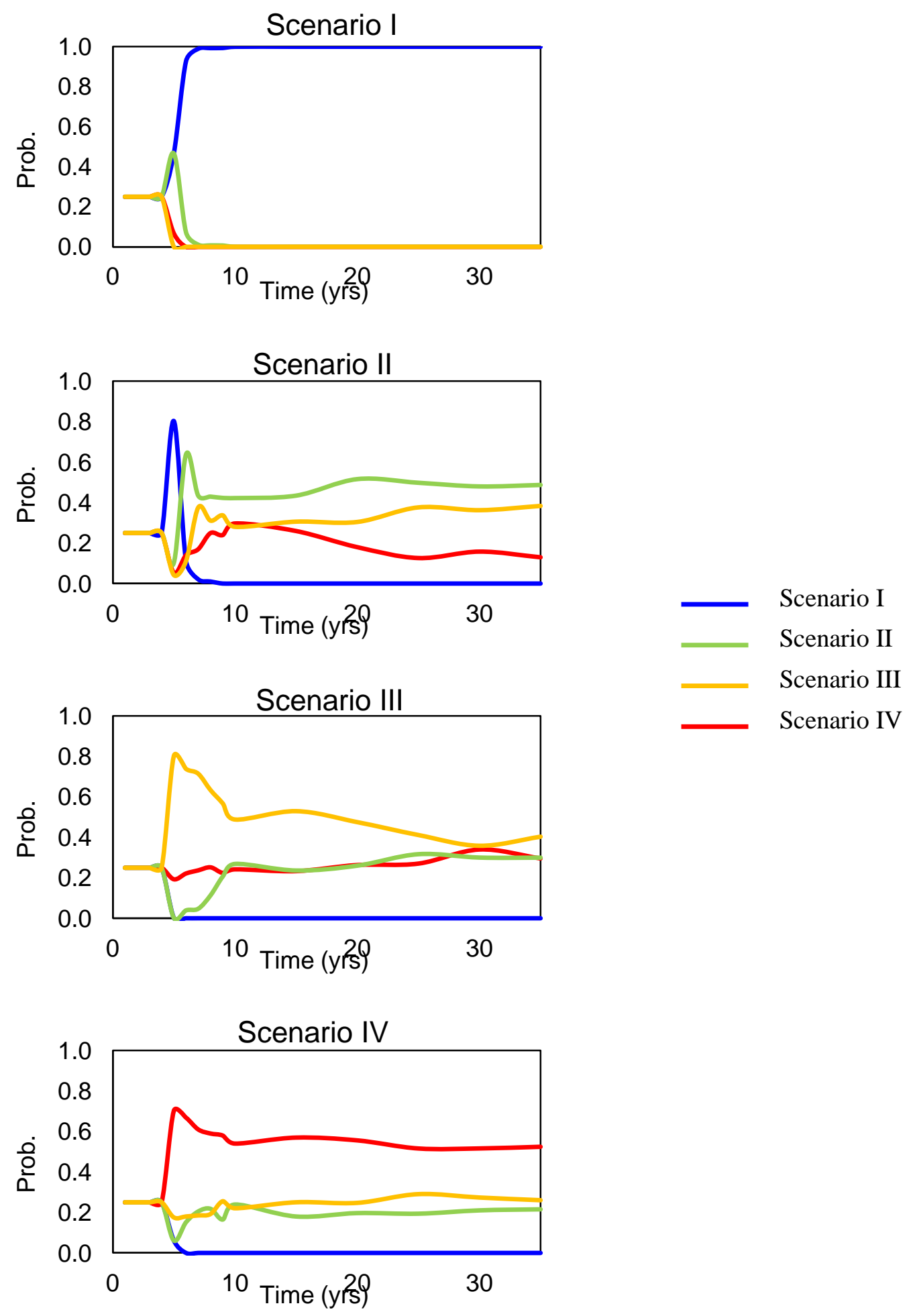

Figure 8: Posterior probabilities inferred for four different caprock fracture scenarios given a true value for a particular scenario (mentioned on top of the plots) until 35 years from the start of injection. Base case caprock thickness of $50 \mathrm{~m}$ and base case injection rate of $1 \mathrm{MT} / \mathrm{yr}$. 

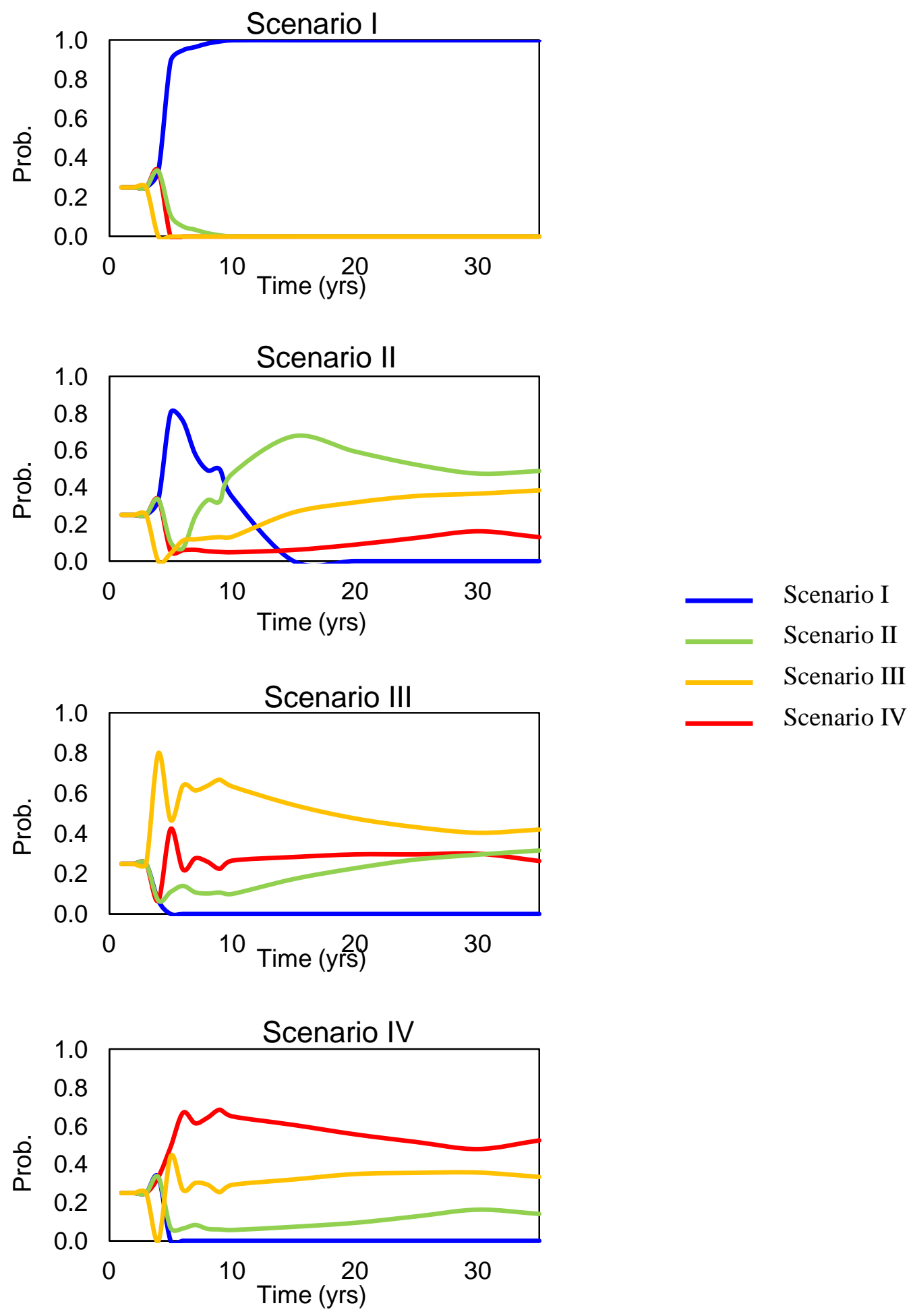

Figure 9: Posterior probabilities inferred for four different caprock fracture scenarios given a true value for a particular scenario (mentioned on top of the plots) until 35 years from the start of injection. Low caprock thickness case of $10 \mathrm{~m}$ and base case injection rate of $1 \mathrm{MT} / \mathrm{yr}$. 

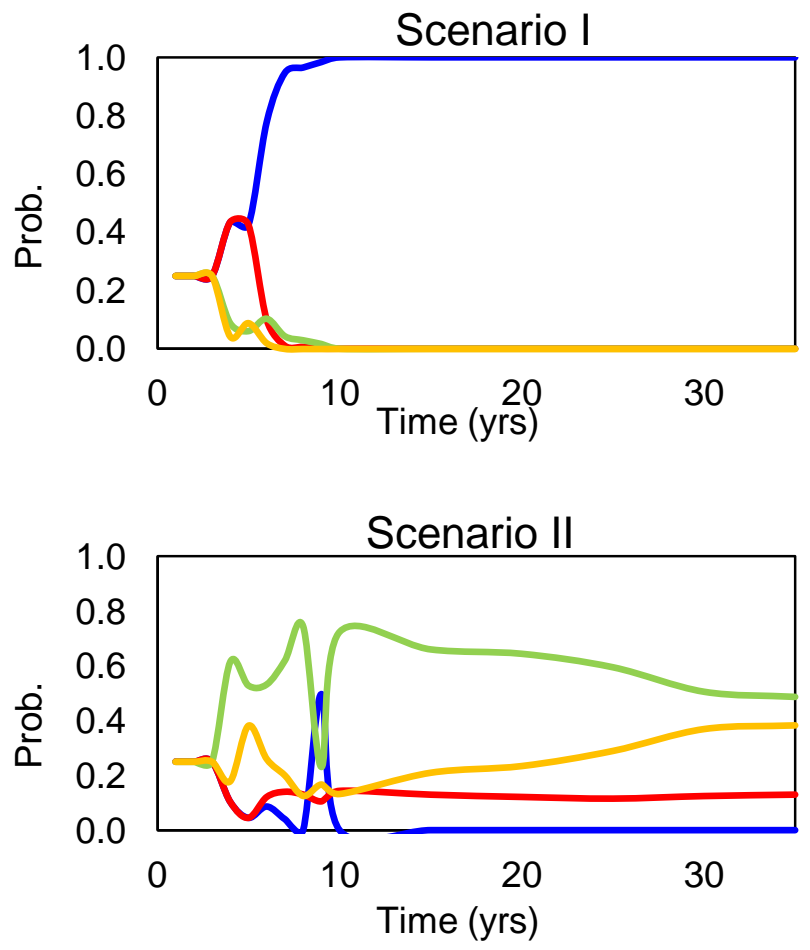

- Scenario I

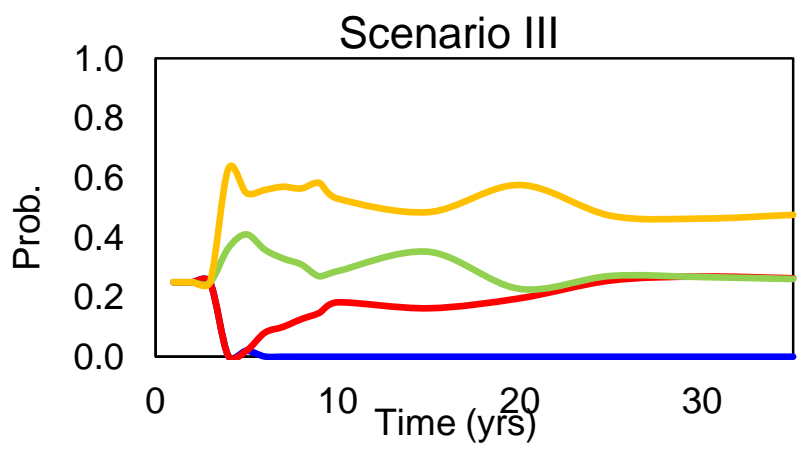

Scenario II

Scenario III

Scenario IV

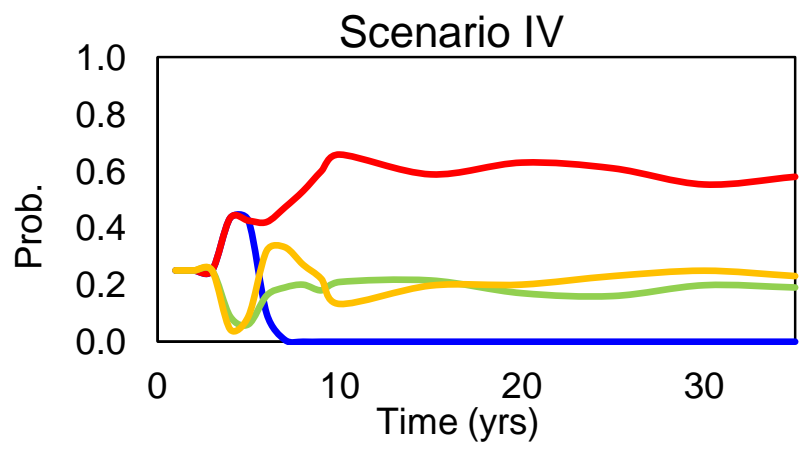

Figure 10: Posterior probabilities inferred for four different caprock fracture scenarios given a true value for a particular scenario (mentioned on top of the plots) until 35 years from the start of injection. High caprock thickness case of $100 \mathrm{~m}$ and base case injection rate of $1 \mathrm{MT} / \mathrm{yr}$. 

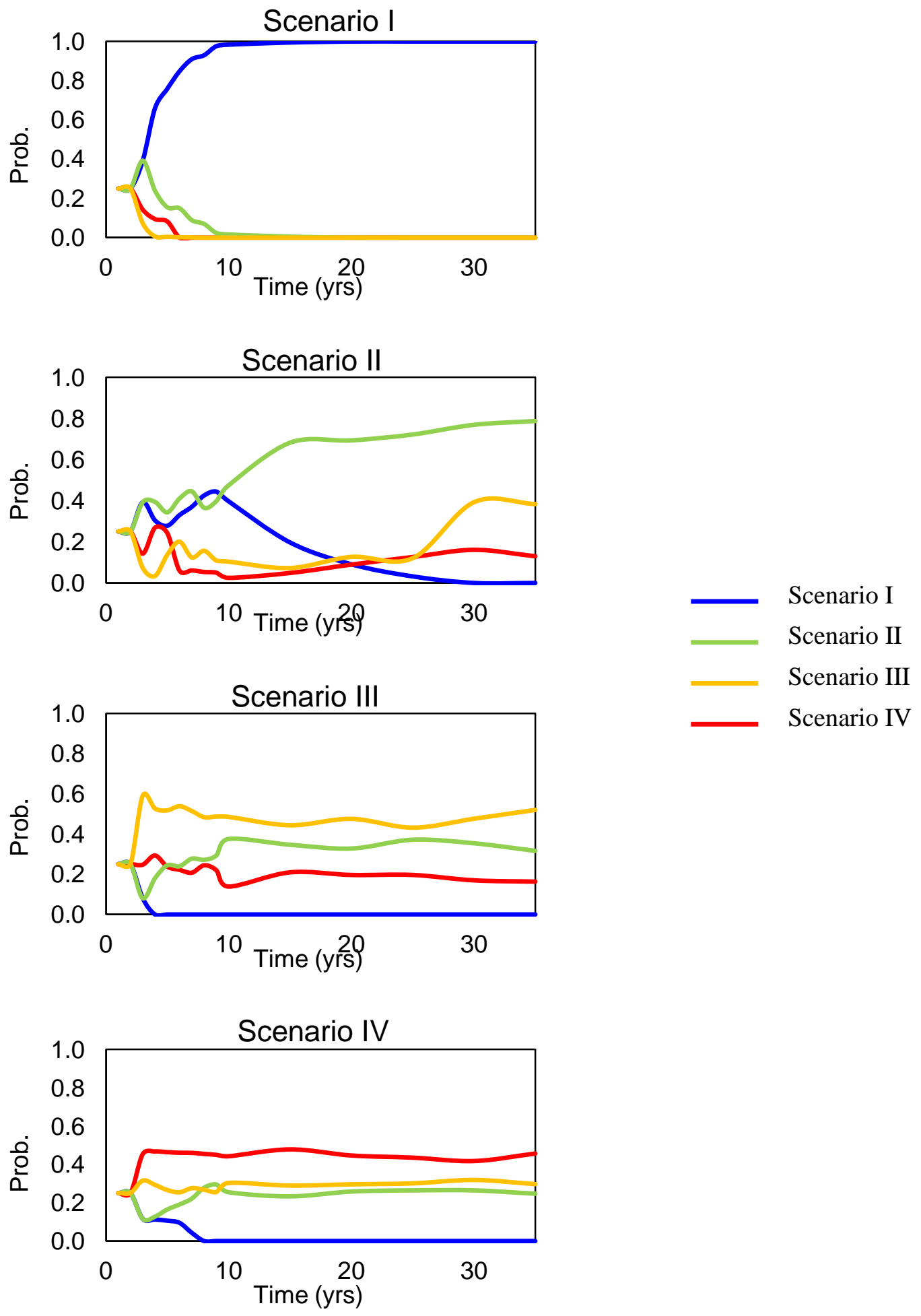

Figure 11: Posterior probabilities inferred for four different caprock fracture scenarios given a true value for a particular scenario (mentioned on top of the plots) until 35 years from the start of injection. Base case caprock thickness of $50 \mathrm{~m}$ and low case injection rate of $0.25 \mathrm{MT} / \mathrm{yr}$. 

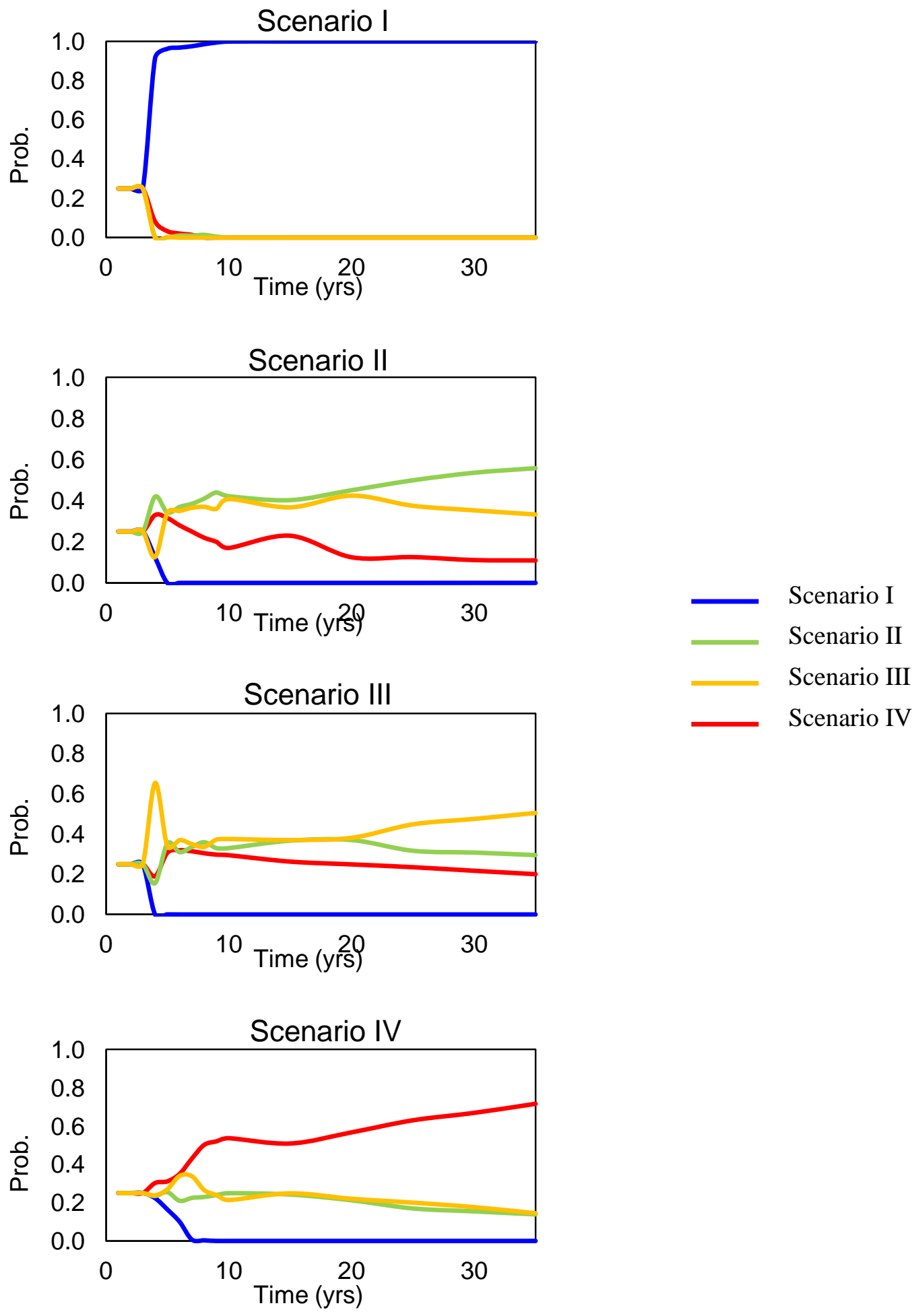

Figure 12: Posterior probabilities inferred for four different caprock fracture scenarios given a true value for a particular scenario (mentioned on top of the plots) until 35 years from the start of injection. Base case caprock thickness of $50 \mathrm{~m}$ and high case injection rate of $5 \mathrm{MT} / \mathrm{yr}$. 

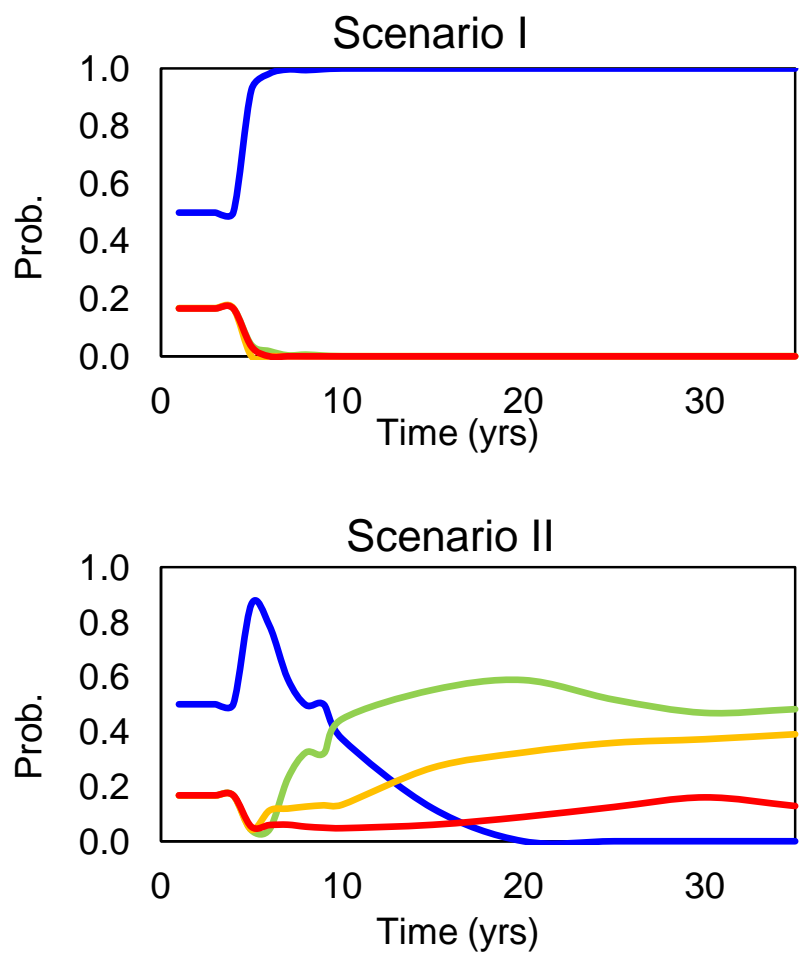

- Scenario I

Scenario II
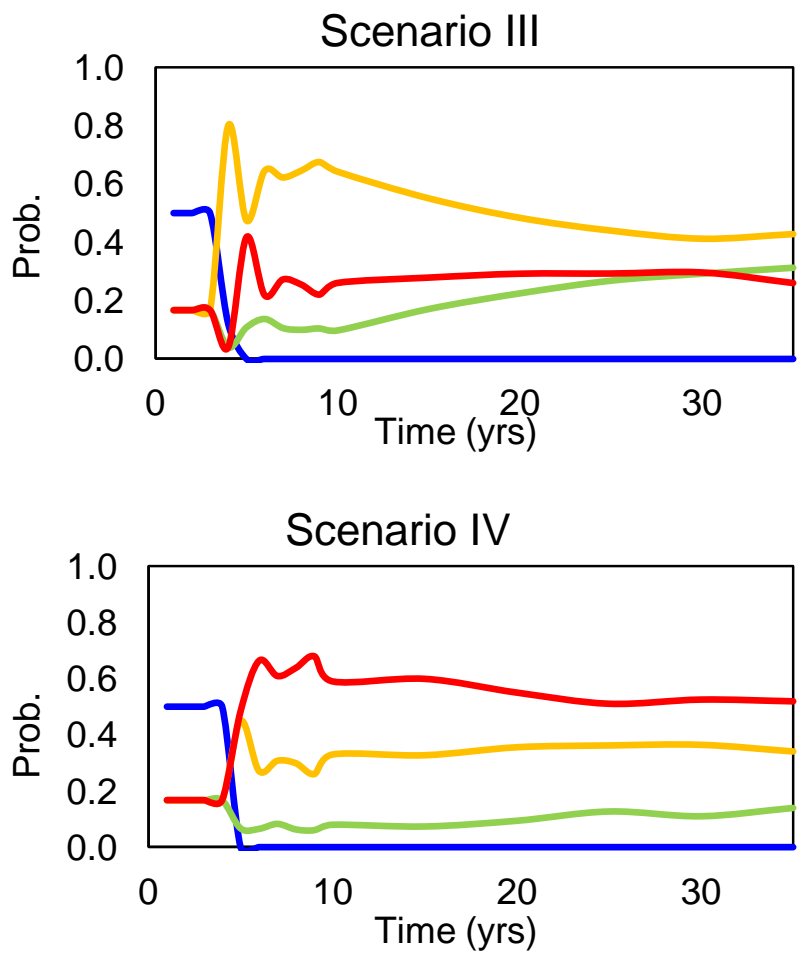

Figure 13: Posterior probabilities inferred for four different caprock fracture scenarios given a true value for a particular scenario (mentioned on top of the plots) until 35 years from the start of injection. Base case caprock thickness of $50 \mathrm{~m}$ and high case injection rate of $1 \mathrm{MT} / \mathrm{yr}$. The prior probability of Scenario I is taken to be 0.50 and that of Scenarios II, III and IV to be 
0.1667 . 
Table 1: Storage reservoir and caprock features

\begin{tabular}{|c|c|c|c|c|c|}
\hline Parameters & Value & & & & \\
\hline Density of rock & $2600 \mathrm{~kg} / \mathrm{m}^{3}$ & & & & \\
\hline Initial pressure at depth $=1000 \mathrm{~m}$ & $10 \mathrm{MPa}$ & & & & \\
\hline Pressure gradient & $10^{4} \mathrm{~Pa} / \mathrm{m}$ & & & & \\
\hline Average temperature at caprock & $50{ }^{\circ} \mathrm{C}$ & & & & \\
\hline Horizontal permeability (storage formation) & $10^{-13} \mathrm{~m}^{2}(0.1 \mathrm{D})$ & & & & \\
\hline Vertical permeability (storage formation) & $10^{-14} \mathrm{~m}^{2}(0.01 \mathrm{D})$ & \multirow{2}{*}{\multicolumn{4}{|c|}{$\begin{array}{l}\text { Table 2: Different fracture scenarios with } \\
\text { their expected effective permeability and } \\
\text { leakage behavior. }\end{array}$}} \\
\hline Salt $(\mathrm{NaCl})$ mass fraction in brine & 0.08 & & & & \\
\hline Porosity (storage formation) & 0.05 & $\begin{array}{l}\text { Capro } \\
\text { ck } \\
\text { scenari } \\
\text { o }\end{array}$ & $\begin{array}{l}\text { Expected } \\
\text { effective } \\
\text { permeabil } \\
\text { ity }\end{array}$ & $\begin{array}{c}\text { Expecte } \\
\text { d } \mathrm{CO}_{2} \\
\text { leakage } \\
\text { magnitu } \\
\text { de }\end{array}$ & $\begin{array}{l}\text { Assumed } \\
\text { prior } \\
\text { probabili } \\
\text { ty }\end{array}$ \\
\hline $\mathrm{CO}_{2}$ residual saturation & 0.1 & I & Low & $\begin{array}{l}\text { Negligib } \\
\text { le }\end{array}$ & 0.25 \\
\hline $\mathrm{CO}_{2}$ injection period & 30 years & II & Very Low & $\begin{array}{l}\text { Moderat } \\
\mathrm{e}\end{array}$ & 0.25 \\
\hline Maximum simulation time & 200 years & III & High & High & 0.25 \\
\hline Domain size & $10 \times 10 \mathrm{~km}$ & IV & $\begin{array}{l}\text { Very } \\
\text { High }\end{array}$ & $\begin{array}{l}\text { Very } \\
\text { High }\end{array}$ & 0.25 \\
\hline Boundary condition & Open boundary & & & & \\
\hline
\end{tabular}


Table 3: Time to no leakage assurance given there is no leakage $\left(T_{\text {no leak }}\right)$

\begin{tabular}{|c|c|c|c|}
\hline Scenario & Seal Thickness & $\begin{array}{l}\text { Injection } \\
\text { Rate }\end{array}$ & $\begin{array}{c}\text { Time to no leak assurance, } \\
\text { Prob }[\text { Scenario } 1 \geq 0.9 \mid \text { Scenario } 1]\end{array}$ \\
\hline \multirow{9}{*}{ I } & \multirow{3}{*}{ Low } & Low & 7 \\
\hline & & Medium & 6 \\
\hline & & High & 4 \\
\hline & \multirow{3}{*}{ Medium } & Low & 8 \\
\hline & & Medium & 6 \\
\hline & & High & 3 \\
\hline & \multirow{3}{*}{ High } & Low & 10 \\
\hline & & Medium & 7 \\
\hline & & High & 5 \\
\hline
\end{tabular}


Table 4: Time to leakage confirmation given there is a leakage $\left(T_{\text {leak }}\right)$

\begin{tabular}{|c|c|c|c|}
\hline Scenario & $\begin{array}{c}\text { Seal } \\
\text { Thickness }\end{array}$ & $\begin{array}{l}\text { Injection } \\
\text { Rate }\end{array}$ & $\begin{array}{c}\text { Time to leakage confirmation, } \\
\text { Prob }[\text { Scenario } 1<0.1 \mid \text { Scenario } j] \text { where } j \neq 1\end{array}$ \\
\hline \multirow{9}{*}{ II } & \multirow{3}{*}{ Low } & Low & 20 \\
\hline & & Medium & 15 \\
\hline & & High & 5 \\
\hline & \multirow{3}{*}{ Medium } & Low & 10 \\
\hline & & Medium & 7 \\
\hline & & High & 4 \\
\hline & \multirow{3}{*}{ High } & Low & 9 \\
\hline & & Medium & 7 \\
\hline & & High & 4 \\
\hline \multirow{9}{*}{ III } & \multirow{3}{*}{ Low } & Low & 3 \\
\hline & & Medium & 4 \\
\hline & & High & 4 \\
\hline & \multirow{3}{*}{ Medium } & Low & 3 \\
\hline & & Medium & 5 \\
\hline & & High & 4 \\
\hline & \multirow{3}{*}{ High } & Low & 3 \\
\hline & & Medium & 4 \\
\hline & & High & 3 \\
\hline \multirow{9}{*}{ IV } & \multirow[t]{3}{*}{ Low } & Low & 6 \\
\hline & & Medium & 5 \\
\hline & & High & 7 \\
\hline & \multirow[t]{2}{*}{ Medium } & Low & 6 \\
\hline & & Medium & 5 \\
\hline & \multirow{4}{*}{ High } & High & 8 \\
\hline & & Low & 8 \\
\hline & & Medium & 6 \\
\hline & & High & 8 \\
\hline
\end{tabular}

